\title{
APEX/SPIN: a free test platform to measure speech intelligibility
}

\author{
Tom Francart Michael Hofmann Jonas Vanthornhout \\ Lieselot Van Deun Astrid van Wieringen Jan Wouters
}

October 31, 2016

To cite this article: Tom Francart, Michael Hofmann, Jonas Vanthornhout, Lieselot Van Deun, Astrid van Wieringen \& Jan Wouters (2016): APEX/SPIN: a free test platform to measure speech intelligibility, International Journal of Audiology

To link to this article: http://dx.doi.org/10.1080/14992027.2016.1247215

\section{Abstract}

Objectives Measuring speech intelligibility in quiet and noise is important in clinical practice and research. An easy-to-use free software platform for conducting speech tests is presented, called APEX/SPIN.

Design The APEX/SPIN platform allows the use of any speech material in combination with any noise. A graphical user interface provides control over a large range of parameters, such as number of loudspeakers, signal to noise ratio and parameters of the procedure. An easy-to-use graphical interface is provided for calibration and storage of calibration values. To validate the platform, perception of words in quiet and sentences in noise were measured both with APEX/SPIN and with an audiometer and CD player, which is a conventional setup in current clinical practice.

Study sample Five normal-hearing listeners participated in the experimental evaluation.

Results Speech perception results were similar for the APEX/SPIN platform and conventional procedures.

Conclusions APEX/SPIN is a freely available and open source platform that allows the administration of all kinds of custom speech perception tests and procedures.

\section{Introduction}

Measures of speech intelligibility in quiet and noise are widely used in clinical practice and research. In clinical practice they are an important part of the basic audiometric test battery (Brandy, 2002; Gelfand, 2001), and they are used for evaluation of the fitting of hearing aids and implants (Dillon, 2001). In research they are often used to investigate the neural mechanisms behind speech intelligibility in noise with normal or impaired hearing, and to evaluate new developments in hearing aids or implants (e.g., Levy et al., 2015).

Speech intelligibility is normally measured using standardised speech materials. These materials usually consist of lists of tokens (words or sentences), which are developed to yield similar 
outcomes across lists and normal-hearing subjects. Typical outcome measures are a percentage correct score or speech recognition threshold (SRT), which is defined as the speech level or signal to noise ratio (SNR) that yields, for example, $50 \%$ intelligibility. To obtain the SRT, a constant stimuli procedure can be used, in which several lists are presented at various SNRs or levels, and the SRT is calculated based on a fit of the performance-intensity function. The use of adaptive procedures can yield a faster estimate of the SRT by conducting only a single list, and varying the SNR or speech level according to an adaptation rule, to efficiently converge to the SRT (Plomp \& Mimpen, 1979).

Over the years, presentation of speech materials has evolved from live voice to playing back from tape or CD. Although this has led to standardization of the materials and calibration, it is still not efficient to use adaptive procedures. Some state-of-the-art audiometers now also provide the option to present speech materials from hard disk, without requiring a CD. However, automatic adaptive procedures are usually not implemented, and it is not straightforward to add extra speech materials and calibrate them correctly and separately from the other materials.

In this paper we present a free and open source test platform to measure speech intelligibility from any personal computer running Windows or GNU/Linux. It is called APEX/SPIN and is built on top of the APEX 3 test platform developed at ExpORL, Dept. Neurosciences (Francart et al., 2008).

We are not aware of any other free and open source platforms for measuring speech intelligibility. There are a number of free platforms for psychophysics, such as PsychoPy (Peirce, 2007), and a number of commercial psychophysics platforms, but they do not provide explicit support for speech testing. There are also a number of commercial platforms for speech testing, such as Rspace (Revit et al., 2007) and Oldenburg Measurement Application (http://hearcom.eu/prof/ DiagnosingHearingLoss/AuditoryProfile/OMABasicSoftware.html), but these are not freely available, nor can they be freely modified by the user.

While it is fairly straightforward to implement a particular speech intelligibility test on the APEX platform, this approach lacks the flexibility of being able to quickly change test parameters and requires some knowledge of APEX. Therefore we developed a plug-in for APEX called APEX/SPIN, which lets the user specify the speech material and a number of other parameters in a graphical user interface (GUI), without requiring any in-depth technical knowledge. In most cases, using APEX/SPIN is as simple as starting up APEX, clicking on the "SPIN" button and setting the parameters in the GUI. In what follows we will give some more information on the principles and possibilities of APEX/SPIN, followed by an experimental comparison between current clinical procedures and APEX/SPIN. Detailed usage instructions can be found in the APEX manual that comes bundled with the software.

\section{Using APEX/SPIN}

\subsection{Concepts}

The function of the APEX platform is described by Francart et al. (2008). In summary, the user specifies an experiment file in the XML format, containing procedural details and references to wav-files containing the audio stimuli. The experiment file is then loaded by APEX, and a user interface is presented to collect subject responses. Finally, APEX can show an analysis of the collected results. APEX/SPIN consists of a GUI that can automatically generate an experiment file for a list of tokens from a speech material and the procedural details specified in the interface. This experiment file is then loaded by APEX and the further course of the experiment and results analysis is identical to normal APEX operation. 


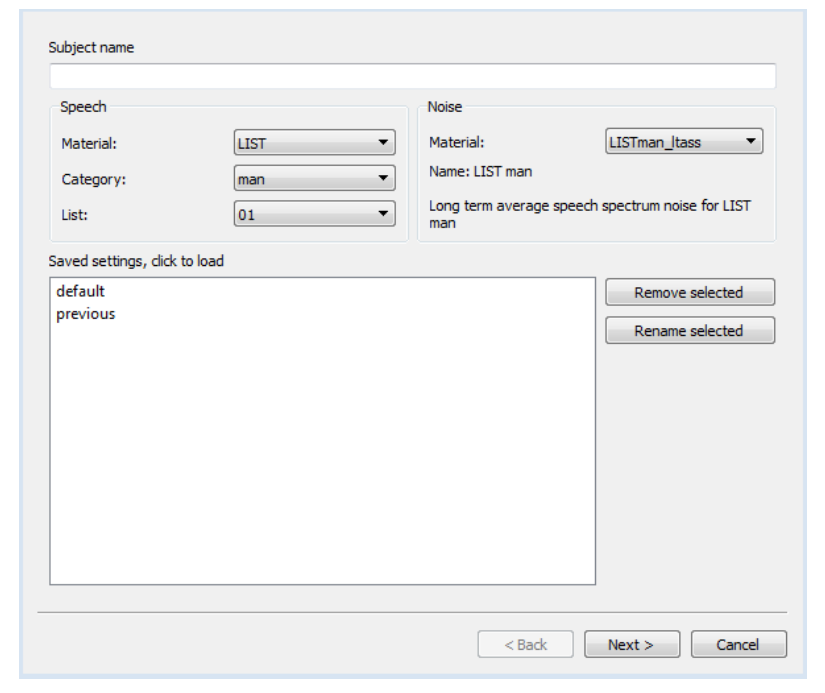

Figure 1: Main window of the APEX/SPIN GUI: selection of speech material, noise and saving of settings.

\subsection{Installation and system requirements}

APEX/SPIN is bundled with the main APEX platform. The software and installation instructions can be found on the APEX website (http://www.kuleuven.be/exporl/apex). On GNU/Linux, one can download the sources and compile them, or request an installer package from the authors. When using APEX/SPIN with speech material provided by ExpORL, the speech material can also be installed from an installer package, without requiring any further configuration.

The system requirements for APEX are minimal, it will run on any modern PC with Windows or GNU/Linux installed. To obtain reliable results, a good quality sound card is required. While nowadays many sound cards integrated with a PC are suitable for conducting speech in noise tests at comfortable levels, for speech in quiet at low levels, a high-quality sound card with large dynamic range (ideally around $120 \mathrm{~dB})^{1}$ is needed. APEX supports any sound card that is supported by the operating system, but best results are generally achieved with sound cards that provide ASIO drivers. Additional to the sound card, high quality headphones are required as well.

\subsection{Functionality}

To illustrate the functionality of APEX/SPIN, in this section, the reader will be guided through using APEX/SPIN, once APEX/SPIN and at least one speech material are installed on the system. To enter the APEX/SPIN GUI, APEX should be started, and the SPIN button should be clicked.

On the first page of the GUI, the speech material to be used is selected (see figure 1). All speech materials consist of lists of tokens (words or sentences), but some materials have additional subdivisions, such as different speakers. Such subdivisions are called categories, which can also be selected in the GUI. In figure 1, list 1 from the LIST speech material is selected, using the male speaker (category "man"). The masker (noise) is in this example the long-term-average-speechspectrum noise supplied with the LIST speech material. Any combination of speech material

\footnotetext{
${ }^{1}$ The dynamic range of modern sound cards is mostly determined by its noise floor. While most sound cards support 24 bit output, many have a noise floor of around $90 \mathrm{~dB}$ below the maximal output level, and therefore an effective dynamic range smaller than the number of bits would suggest.
} 


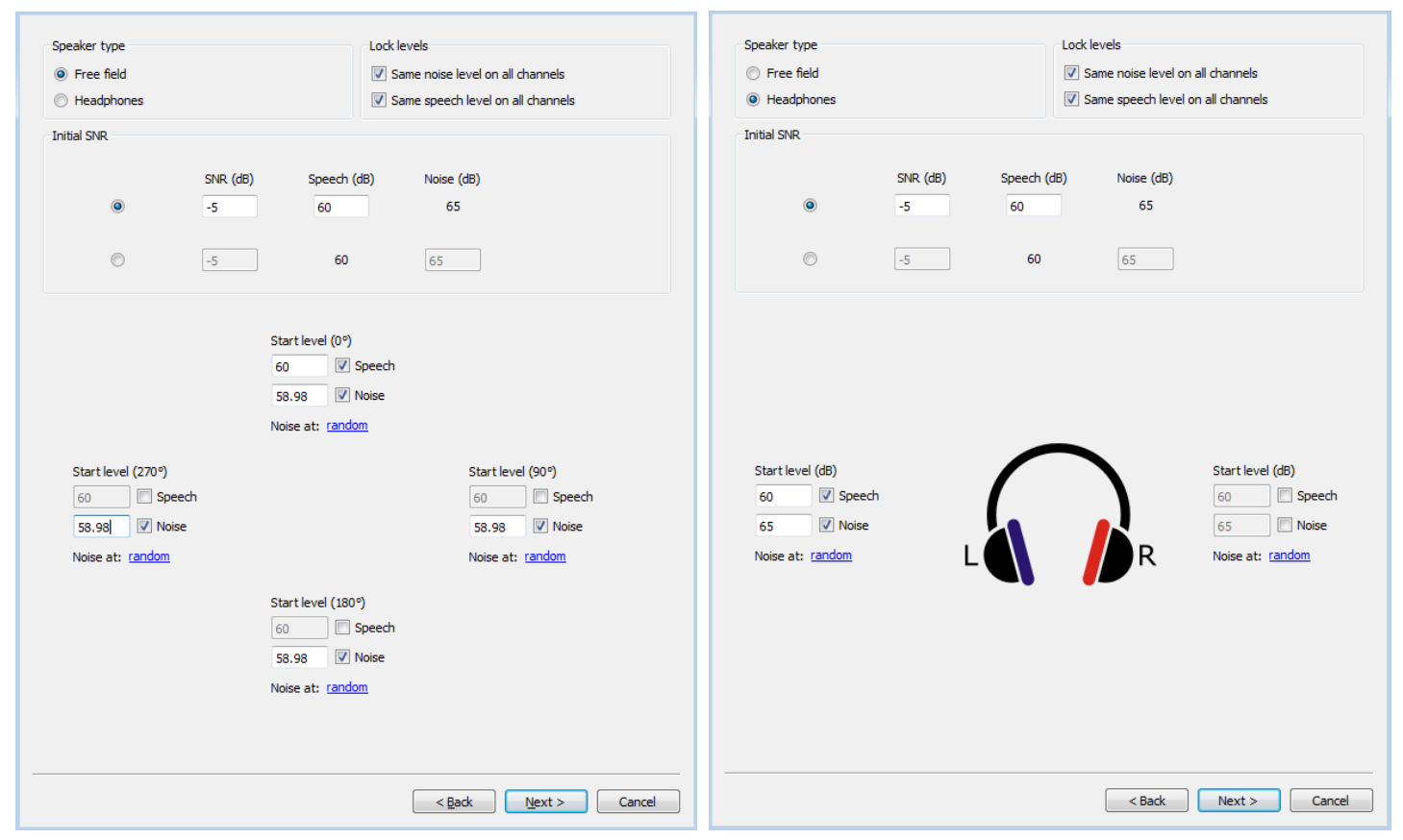

Figure 2: Loudspeaker selection: if "speaker type" is set to free field, the left dialog is shown. If "speaker type" is set to headphones, the right dialog is shown. For each loudspeaker or ear, speech and noise can be turned on or off using the check boxes. If the overall SNR or level is specified, the individual levels will be calculated automatically. The "random" link next to each loudspeaker can be used to indicate that the noise should be started at a random place in the provided wav file.

and noise can be freely selected. Custom speech materials or noises can be added (see manual for the procedure). Below the speech material selection fields, one has the possibility to save a set of SPIN settings for later use. For instance, if one often uses the LIST material in noise under headphones with an adaptive procedure, these settings can be saved and loaded later on with a single click.

On the second page (Speakers), the loudspeakers or headphones to be used can be specified (see figure 2). First, either free field or headphone stimulation has to be selected. For headphone or single-loudspeaker free field stimulation, the overall speech and noise level obviously correspond to the speech and noise level specified for one ear (right panel of Figure 2) or for one loudspeaker. In free field mode, one can specify the overall speech and noise level, and then speech and noise levels for the individual loudspeakers are automatically calculated, by adding power in linear units (assuming that the noise is uncorrelated across loudspeakers) and converting back to decibels. An example is shown in the left panel of Figure 2. Alternatively, speech and noise levels can be specified separately for each ear or loudspeaker.

On the third page, procedural parameters can be entered (see figure 3). For constant procedures there are no parameters, but for adaptive procedures, the step size (in $\mathrm{dB}$ ) can be specified, and there is an option to repeat the first token until a correct answer is given (Plomp \& Mimpen, 1979) in order not to use too many tokens at SNRs far from the SRT. On the top right, the user can indicate whether the speech level should be adapted, in which case the noise level will be fixed throughout the experiment, or whether the noise level should be adapted, in which case the speech level will be fixed. A fixed noise level is often used when testing noise suppression algorithms. A fixed speech level is often used when the speech needs to fit within a hearing-impaired subject's limited dynamic range. The starting value of the parameter that is adapted (speech or noise level) is determined by the values entered in the Speakers tab. 




Figure 3: GUI for setting parameters of the adaptive procedure. For the constant procedure, no additional parameters can be specified.

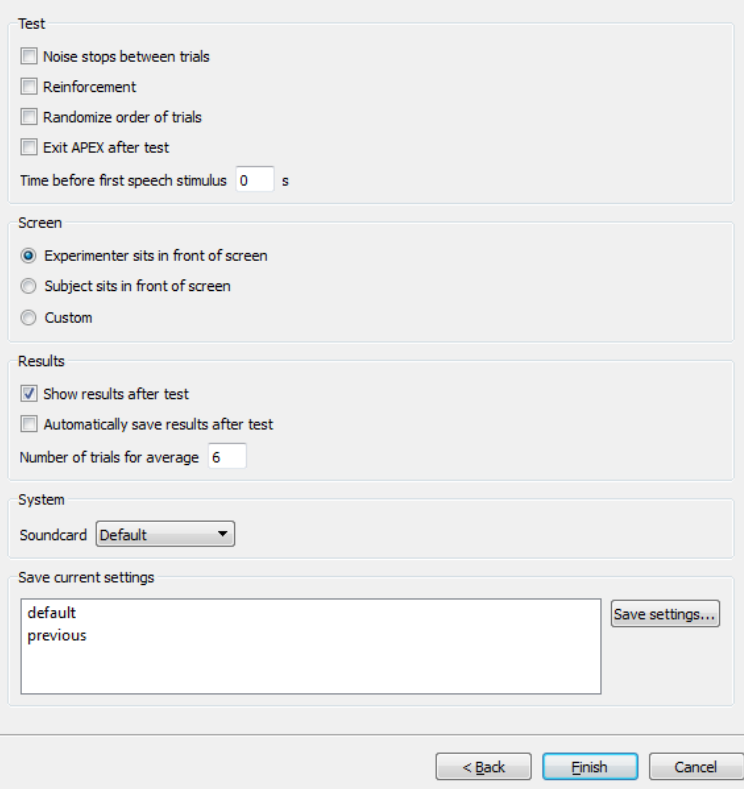

Figure 4: GUI for setting various options 


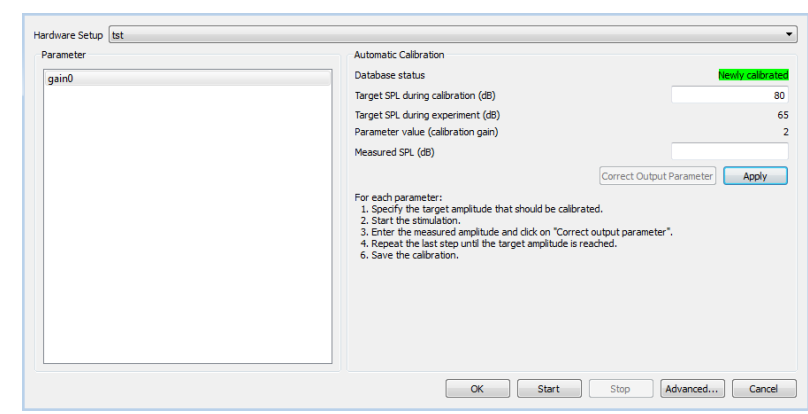

Figure 5: Calibration dialog. In this example there is only one loudspeaker and its level has been calibrated (indicated by the green message).

Finally, on the fourth page, various options can be specified (see figure 4). Most options are self-explanatory. A detailed description can be found in the APEX manual.

When all tabs have been completed, the user can press the "create experiment" button. An experiment file will then be generated, and APEX will query where it should be saved on disk. If the experiment file is stored together with the results file, all experimental parameters can be retrieved later on.

\subsection{Calibration}

For calibration, the APEX calibration infrastructure is used (Francart et al., 2008). In summary, during calibration the value of an APEX parameter (e.g., gain of the first channel of the sound card) is linked to a sound pressure level, measured with a sound level meter, entered by the user. Calibration values are stored in a database for each combination of hardware setup and calibration profile. The hardware setup is a string that describes the external hardware attached to the computer, for example, the type of sound card or type of headphones. It is shown at the top of the calibration dialog (see figure 5). With APEX/SPIN, a different calibration profile is generated for each combination of speech material and noise. The noise is used as the calibration stimulus. In most cases this will be the long term average speech spectrum noise accompanying the speech material. When no calibration value is found in the database for the current hardware setup and calibration profile, APEX will automatically present the calibration window. Thereafter, the user can recalibrate at any time using the calibration menu.

\subsection{Adding speech materials and noises}

Our lab provides a number of speech materials in a format readily usable with APEX/SPIN, including the Leuven Intelligibility Sentence Test (LIST) and Leuven Intelligibility Number Test (LINT) (van Wieringen \& Wouters, 2008), the Leuven Intelligibility Sentence test with male speaker (LIST-m, Jansen et al., 2014), the French Intelligibility Sentence Test (FIST, Luts et al., 2008) and French Matrix test (FrMatrix, Jansen et al., 2012), the word lists of the "Nederlandse Vereniging voor Audiologie" (NVA-lists, Wouters et al., 1994), the sentence lists of the "Vrije Universiteit Amsterdam" (VU-lists, Versfeld et al., 2000), and the Hearing in Noise Test (HINT, Nilsson et al., 1994). Alternatively, users can easily add their own speech materials and noises. This involves creating an XML file with the required information. Details can be found in the APEX manual. 




Figure 6: Example display of results of an adaptive procedure after conducting an experiment. The adaptive staircase is shown, as well as the SRT, in this case calculated as the mean of the last 6 trials (including a virtual trial, calculated based on the last result).

\subsection{Customising}

APEX/SPIN has some settings that cannot be set in the GUI, but can be configured by advanced users (in the spin.xml file). It concerns for example the speaker setup, what is displayed on the screen, the test procedure, or the way results are presented or analysed. If further customisations are required that are not available in the GUI or spin.xml file, there are two remaining options: editing the experiment file generated by APEX/SPIN, or changing the source code of APEX/SPIN itself, which is freely available. APEX uses standard HTML/JavaScript technology to display the results, which allows for customisation of the way results are analysed and shown.

\subsection{Processing results}

If the option "Show results after test" is ticked in the Options tab of the APEX/SPIN dialog, an analysis of the results will be shown right after the experiment (see figure 6). From this analysis, one can simply note the required value (e.g., the SRT or percentage correct), print the results, export to PDF, or copy the raw data to another program. When a results file is opened in APEX at a later stage, the same analysis is shown. The full results file should be stored such that additional information that may be required at a later time for further analysis, such as reaction times, is still available.

Alternatively to copying the results manually, one can automatically collect the results. For example, data can be exported to the comma separated values (CSV) format or can be processed directly with the APEX Matlab Toolbox or APEX R Toolbox that come bundled with APEX.

\section{Evaluation of APEX/SPIN}

\subsection{Experimental and physical validation}

The reliability of the APEX/SPIN interface was evaluated in a group of 5 young normal-hearing listeners (between 20 and 30 years of age). All listeners were tested with two setups. In one setup, APEX/SPIN ran on a Windows PC with RME soundcard and HDA200 headphones. In the second setup, a MADSEN audiometer was used together with a CD player and HDA200 

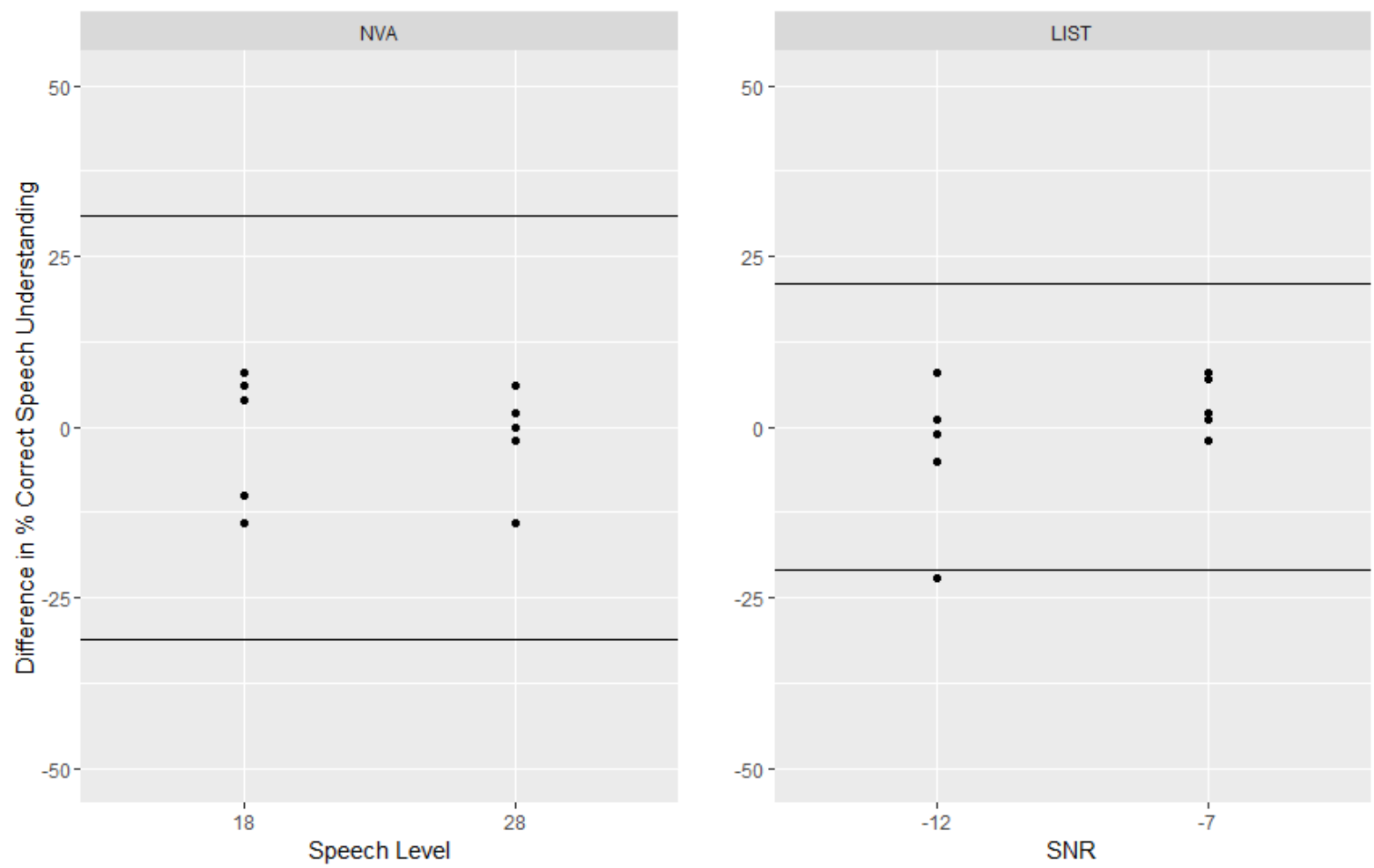

Figure 7: Difference in percentage correct speech perception between both procedures, i.e. PC with APEX/SPIN versus audiometer with CD. Results are shown for NVA words (left panel) versus LIST sentences (right panel) and for low and high intensities or signal-to-noise ratios (SNRs). The horizontal black lines indicate the range of test-retest differences. 
headphones, which is a conventional clinical setup. Both setups were calibrated with a Brüel \& Kjaer sound level meter (2260) and artificial ear (4153).

Both NVA words in quiet (Bosman \& Smoorenburg, 1995; Bosman et al., 1995) and LIST sentences in noise (van Wieringen \& Wouters, 2008) were used. Participants listened to two lists of each speech material for each setup and for two intensity levels or signal-to-noise ratios (SNRs). Each list of words or sentences was presented at a fixed intensity. The intensities were chosen to cover both the lower and the upper region of the function relating percentage correct to intensity. For the NVA, speech levels were 18 and $28 \mathrm{~dB}$ SPL. For the LIST, SNRs were -12 and $-7 \mathrm{~dB}$, with a fixed noise level of $65 \mathrm{~dB}$ SPL. Tests were randomized for speech lists, setup and presentation level across subjects.

Results of the speech perception experiment are shown in figure 7. This figure displays the difference in percentage correct speech perception between both procedures, i.e. PC with APEX/SPIN versus audiometer with CD. Results are shown for NVA words (left panel) versus LIST sentences (right panel) and for low and high intensities or SNRs. The horizontal black lines indicate the range of test-retest differences, i.e. the maximum absolute difference between test and retest score across subjects. These lines show that speech perception with a limited number of items presented at a fixed level is prone to a rather large variability. The differences in results between both setups, however, are well within the test-retest range (except for one outlier), demonstrating the reliability of the APEX/SPIN setup.

\subsection{User experience}

Experience with APEX/SPIN has been gained in our lab, and in the ENT department of University Hospitals Leuven and the related rehabilitation center MUCLA. In a clinical context, for presentation of standardized words or sentences at a fixed level with the corresponding noise, the audiometer with CD player or with integrated speech lists are considered to be convenient procedures. The advantage of the APEX/SPIN interface particularly appears when using adaptive procedures, when using custom speech and noise materials, or when testing with more than two loudspeakers. The conventional setups require pausing, changing the level, writing down the response after each item, and calculating the result manually. This procedure is time consuming, requires a high level of concentration, and is extremely prone to errors. Automatic adaptation of the level and processing of the results with APEX/SPIN overcomes these problems.

APEX/SPIN has now been satisfactorily used in all speech testing in our lab (ExpORL, Dept. Neurosciences, KU Leuven) over a number of years. In an experimental setting, a noted advantage of the APEX/SPIN interface is combination of usability - no programming required and flexibility, including the possibility of adding different speech and noise materials and setting calibration values differently and therefore appropriately for each material.

\section{Discussion and conclusions}

APEX/SPIN is a platform to easily and quickly perform speech intelligibility tests in quiet and noise. It is available for free and open source. We distribute various speech materials that can be directly used with APEX/SPIN, but additional materials can easily be added. APEX/SPIN has been used successfully in various studies in our lab so far, and thoroughly validated in the course of those studies. It can be freely downloaded from our website http: //www.kuleuven.be/exporl/apex, and the source code is available under the General Public License $\mathrm{v} 2^{2}$.

\footnotetext{
${ }^{2}$ http://www.gnu.org/licenses/gpl-2.0.html
} 
Advantages of using APEX/SPIN over a custom implementation or audiometer-based setup are that (1) speech intelligibility tests in quiet and noise can be quickly and easily conducted, without any programming, with extensive control over several parameters, (2) any combination of speech material and noise can be used, in any spatial configuration, and (3) an easy-to-use graphical user interface is available for calibration and storage of calibration data.

\section{Acknowledgments}

We are grateful to our colleagues at ExpORL, Dept. Neurosciences, KU Leuven for providing valuable feedback on earlier versions of the software. We thank Job Noorman for helping with the programming. Financial support for this project is provided by the KU Leuven Special Research Fund under grant OT/14/119.

\section{References}

Bosman A.J. \& Smoorenburg G., 1995. Intelligibility of Dutch CVC syllables and sentences for listeners with normal hearing and three types of hearing impairment. Audiology, 34, 260-284.

Bosman A.J., Wouters J. \& Damman W., 1995. Realisatie van een cd voor spraakaudiometrie in Vlaanderen. Logop en Foniatr, 9, 218-225.

Brandy W.T., 2002. Speech Audiometry. In J. Katz, editor, Handbook of Clinical Audiology, chapter 7, pp. 96-110. 5th edition.

Dillon H., 2001. Assessing the outcomes of hearing rehabilitation. In H. Dillon, editor, Hearing aids, chapter 13, pp. 349-369.

Francart T., van Wieringen A. \& Wouters J., 2008. APEX 3: a multi-purpose test platform for auditory psychophysical experiments. J Neurosci Methods, 172(2), 283-93.

Gelfand S.A., 2001. Speech Audiometry. In S.A. Gelfand, editor, Essentials of Audiology, chapter 8, pp. 257-290. 2nd edition.

Jansen S., Koning R., Wouters J. \& van Wieringen A., 2014. Development and validation of the Leuven intelligibility sentence test with male speaker (LIST-m). Int J Audiol, 53(1), 55-9.

Jansen S., Luts H., Wagener K.C., Kollmeier B., Del Rio M., Dauman R., James C., Fraysse B., Vormès E., Frachet B., Wouters J. \& van Wieringen A., 2012. Comparison of three types of French speech-in-noise tests: a multi-center study. Int J Audiol, 51(3), 164-73.

Levy S.C., Freed D.J., Nilsson M., Moore B.C.J. \& Puria S., 2015. Extended High-Frequency Bandwidth Improves Speech Reception in the Presence of Spatially Separated Masking Speech. Ear and hearing, 36(5), e214-24.

Luts H., Boon E., Wable J. \& Wouters J., 2008. FIST: a French sentence test for speech intelligibility in noise. Int $J$ Audiol, 47(6), 373-4.

Nilsson M., Soli S.D. \& Sullivan J.A., 1994. Development of the Hearing in Noise Test for the measurement of speech reception thresholds in quiet and in noise. J Acoust Soc Am, 95(2), $1085-1099$.

Peirce J.W., 2007. PsychoPy-Psychophysics software in Python. J Neurosci Methods, 162(1-2), $8-13$. 
Plomp R. \& Mimpen A.M., 1979. Improving the reliability of testing the speech reception threshold for sentences. Audiology, 18(1), 43-52.

Revit L., Killion M. \& Conley C., 2007. Developing and Testing a Laboratory Sound System That Yields Accurate Real-World Results. The Hearing Review.

van Wieringen A. \& Wouters J., 2008. LIST and LINT: sentences and numbers for quantifying speech understanding in severely impaired listeners for Flanders and the Netherlands. Int $J$ Audiol, 47(6), 348-55.

Versfeld N.J., Daalder L., Festen J.M. \& Houtgast T., 2000. Method for the selection of sentence materials for efficient measurement of the speech reception threshold. J Acoust Soc Am, 107(3), 1671-84.

Wouters J., Damman W. \& Bosman A.J., 1994. Vlaamse opname van woordenlijsten voor spraakaudiometrie. Logop informatiemedium van Vlaam Ver voor Logop, 7(6), 28-34. 